\title{
Effects of Denosumab Treatment during Early Pregnancy - A Case Report
}

Fumihiro Isobe $^{1,2}$, Yukio Nakamura ${ }^{1^{*}}$, Mikio Kamimura ${ }^{3}$, Shigeharu Uchiyama $^{1}$ and Hiroyuki Kato ${ }^{1}$

${ }^{1}$ Department of Orthopaedic Surgery, Shinshu University School of Medicine, Asahi 3-1-1, Japan

${ }^{2}$ Department of Orthopaedic Surgery, Aizawa Hospital, Honjo 2-5-1, Japan

${ }^{3}$ Center of Osteoporosis and Spinal Disorders, Kamimura Orthopaedic Clinic, Ipponmatsu 595-17, Japan

"Corresponding author: Yukio Nakamura, , MD, PhD, Department of Orthopaedic Surgery, Shinshu University School of Medicine, Asahi 3-1-1, Matsumoto 390-8621, Japan, Tel: +81-263-37-2659; Fax: +81-263-35-8844; E-mail: yxn14@aol.jp

Rec date: May 18, 2016; Acc date: Jun 06, 2016; Pub date: Jun 10, 2016

Copyright: (C) 2016 Isobe F, et al. This is an open-access article distributed under the terms of the Creative Commons Attribution License, which permits unrestricted use, distribution, and reproduction in any medium, provided the original author and source are credited.

\begin{abstract}
Background: Osteoporotic treatment during pregnancy is controversial. Denosumab, a fully human monoclonal antibody against receptor activator of nuclear factor-kB ligand (RANKL), is a potent new bone resorptive inhibitory drug for osteoporotic treatment. There has been no report on the effectiveness or adverse effects of denosumab treatment in pregnant osteoporotic patients.
\end{abstract}

Case: We encountered a young woman with osteoporosis due to idiopathic menstrual irregularity. Prior to therapy, we confirmed that she would not become pregnant during the treatment period. A single dose of denosumab, was given to the woman who, despite becoming pregnant within a week afterwards, completed delivery without any serious complications for mother or child. We regularly examined the values of bone chemical markers during and after pregnancy along with bone mineral density (BMD) before treatment and after giving birth. Marker values were improved at 6 months after the initial denosumab injection.

Conclusion: These results suggested that BMD and bone turnover marker values were improved at 6 months after denosumab administration during unintended early pregnancy in a young woman with osteoporosis. To our knowledge, although contraindicated in pregnancy, this is the first report of denosuamb use in pregnancy. Our purpose was to demonstrate a case in which it was used in such a context without adverse effects.

Keywords: Pregnancy; Osteoporosis; Denosumab

\section{Case Presentation}

A 25-year-old Japanese woman sought consultation at our institution to determine the requirement of osteopenic or osteoporotic treatment. Her height was $158 \mathrm{~cm}$, body weight was $46 \mathrm{~kg}$, and body mass index was $18.4 \mathrm{~kg} / \mathrm{m}^{2}$ at the time. She was single and not pregnant. Although she had no history of bone metabolic diseases and had never taken medicine affecting bone metabolism, she had earlier experienced a fragility fracture of the third lumbar (L) vertebra. She had also taken Chinese herbal medicine from 21-23 years of age for idiopathic menstrual irregularity starting from the age of 17 years. She previously visited our institution complaining of pallor and psychroesthesia when both hands were cold at age 18, for which a certified internist ruled out the possibility of obvious collagen disease.

At presentation, plain radiographs showed mild osteoarthritis in the left hip joint (data not shown). Bone mineral density (BMD) was measured using a DXA fan-beam bone densitometer (Lunar Prodigy; GE Healthcare, Waukesha, WI, USA) at the L1-4 levels of the posteroanterior spine (L-BMD) and bilateral hips (H-BMD) immediately before and 1 year after denosumab administration, and standard biochemical analyses were performed regularly as described below. Table 1 summarizes the BMD and bone turnover marker results before and after treatment. Prior to denosumab therapy, we confirmed the last menstruation to be within 1 month beforehand.

\begin{tabular}{|c|c|c|}
\hline \multicolumn{2}{|c|}{ Biochemical markers before treatment } & Normal range \\
\hline \multicolumn{3}{|c|}{ Serum } \\
\hline Calcium (mg/dL) & 9.5 & $8.7-10.3$ \\
\hline Phosphorus (mg/dL) & 3.3 & $2.5-4.6$ \\
\hline TRACP-5b (mU/dL) & 366 & $120-420$ \\
\hline $\mathrm{BAP}(\mu \mathrm{g} / \mathrm{L})$ & 13.1 & $3.7-20.9$ \\
\hline \multicolumn{3}{|c|}{ Urine } \\
\hline NTX (nM BCE/mM Cr) & 45 & $<35.5$ \\
\hline \multicolumn{3}{|c|}{ BMD before and after treatment } \\
\hline & Before & After \\
\hline Lumbar spine BMD (g/cm²) & 0.874 & 0.891 \\
\hline T Score & -2.1 & -1.9 \\
\hline Right femoral neck BMD $\left(\mathrm{g} / \mathrm{cm}^{2}\right)$ & 0.674 & 0.692 \\
\hline T Score & -2.2 & -2 \\
\hline Left femoral neck BMD $\left(\mathrm{g} / \mathrm{cm}^{2}\right)$ & 0.664 & 0.659 \\
\hline T Score & -2.2 & -2.3 \\
\hline
\end{tabular}

Table 1: Patient characteristics before and after denosumab treatment. 
Our nursing staff carefully ascertained that the woman was not planning to become pregnant during treatment and clearly explained the possible adverse effects of denosumab on babies in the perinatal pregnancy period along with the pros and cons of various osteoporotic drugs. Since the patient could not regularly visit us due to her location and denosumab had relatively less complications as compared with bisphosphonates (BPs) and other treatments, she selected denosumab treatment. However, soon after a single denosumab administration $(60$ mg; normal dosing), it was discovered that she had become pregnant. Based on discussion with the patient, the onset of pregnancy was estimated to have occurred 4 days after denosumab was given. Thus, denosumab treatment was halted since the medication was feared to cause adverse effects. Calcium and vitamin D were also stopped due to possible detrimental effects, such as constipation.

The changes in several biochemical marker values are shown in Figures 1-3. Serum corrected calcium and phosphate remained within normal ranges up to a year following denosumab treatment. Among bone resorption markers, urinary N-terminal telopeptide (NTx) and serum tartrate-resistant acid phosphatase 5b (TRACP-5b) were markedly decreased at 1 month after drug administration, returned to baseline levels at months 7-8, peaked, and then decreased over the final months of the study period. The bone formation markers bone alkaline phosphatase (BAP) and serum C-telopeptide of type I collagen propeptide (PINP) decreased slightly during the initial 1-2 months following denosumab administration and then spiked at or around the time of delivery. The values of $1,25(\mathrm{OH})_{2} \mathrm{D}_{3}$ gradually increased until 7 months after drug administration and then decreased. Serum concentrations of $25(\mathrm{OH}) \mathrm{D}$ were mildly increased at 8 months but had returned to baseline levels by the study end point. Whole parathyroid hormone (PTH) values tended to increase after an initial drop at 2 months. Serum pentosidine had decreased at 5 months, increased at months 8-11, and decreased at month 12 . H-BMD and L-BMD were increased a year after denosumab administration (Table 1). (a)

\section{(mg/dL) Serum corrected calcium}

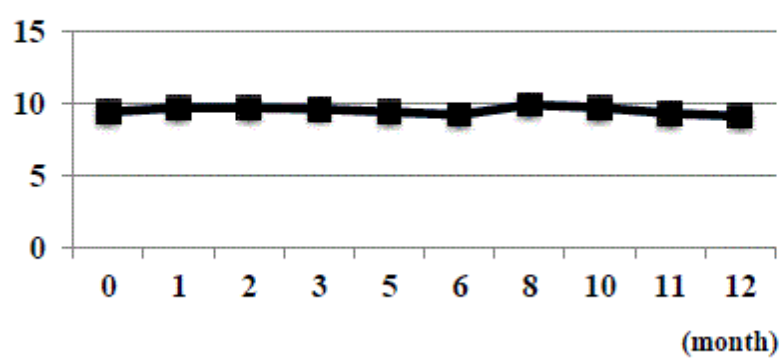

(b)

$(\mathrm{mg} / \mathrm{dL}) \quad$ Serum phosphate

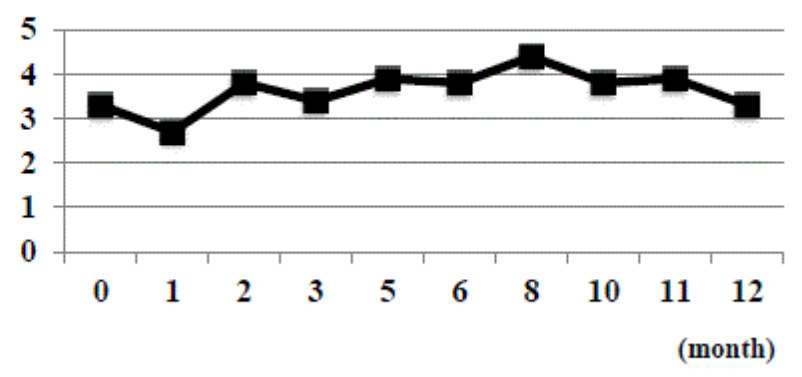

Figure 1: Changes in the values of serum corrected calcium (a) and phosphate (b) during 1 year after denosumab treatment.

The patient's son was delivered by cesarean section at 36 weeks of gestation without any apparent complications during the pregnancy. The baby weighed 2,392 $\mathrm{g}$ and his APGAR SCORE was 7/7. However, the infant was hospitalized due to unstable respiratory status from transient neonatal tachypnea and slightly premature birth. Although there was 1 remarkable episode of decreased $\mathrm{SpO}_{2}$, such events gradually improved. Feeding performance was also low for several days and serum blood sugar level hovered around $50 \mathrm{mg} / \mathrm{dl}$, but these ameliorated soon thereafter.

At the present time 12 months after birth, the boy's height and weight are both comparable to those of normal growth curves.

The current study was approved by our Institutional Ethics Committee at Shinshu University School of Medicine, and informed consent was obtained from the patient for publication. All methods were carried out in accordance with the approved guidelines.

\section{Discussion}

Receptor activator of nuclear factor-kB ligand (RANKL) is a cytokine that is essential for osteoclast differentiation, activation, and survival [1]. Denosumab, a fully human monoclonal antibody against RANKL shown to selectively inhibit osteoclastogenesis, was approved for use in Japan in 2013. As severe osteoporosis in RANKL transgenic mice was reversed by denosumab administration [2], RANKL appears to be an ideal target for osteoporosis treatment. Boyce et al. reported that in utero denosumab exposure in monkeys produced an osteoclastpoor osteopetrotic-like skeletal phenotype [3]. They also described that stillborn birth rate and infant skeletal abnormalities increased after denosumab treatment in monkeys [3]. In addition, RANKL knockout mice exhibited severe osteopetrosis with a complete absence of osteoclasts, suggesting that RANKL was an essential factor for osteoclastogenesis [4]. To our knowledge, there have been no reports or clinical trials on the effects of denosumab treatment during early pregnancy in humans.

The advocacy of BP administration during pregnancy is controversial. Although BPs are known to cross the placental barrier and affect fetal serum calcium levels and growth, there have been no reports of congenital abnormalities associated with BPs in animal teratology studies [5,6]. Sanz et al. analyzed 51 pregnancies in which the subjects had been taking BPs shortly before or during the initial weeks of pregnancy and described no major anomalies in the neonates. However, there is a general consensus that this treatment should be avoided during gestation [7].

In pregnancy, calcium is shunted from the maternal circulation to that of the fetus in order to mineralize the developing fetal skeleton [8]. Approximately $80 \%$ of circulating calcium is considered to transfer 
from mother to fetus during late pregnancy. Thus, it is considered that fetus receives sufficient calcium for proper development [9], and that the maternal adaptations to meet this demand start early in gestation. In support of this, abundant vitamin $\mathrm{D}$ is produced in the placenta $[10,11]$, and $1,25(\mathrm{OH})_{2} \mathrm{D}_{3}$ levels increase early in pregnancy and remain approximately twice as high as pre-pregnancy levels due to PTH-independent up-regulation of 1a-hydroxylase in the kidneys. Longitudinal measurements of intact PTH levels have revealed decreases to a low-to-normal range during the first trimester of pregnancy, with subsequent increases to a mid-normal range by full term [12]. Calcium levels have been shown to decrease to a low-to- normal range during early pregnancy. In total, approximately $30 \mathrm{~g}$ of calcium transfers from the mother to the fetus via the placenta. After delivery, maternal calcium is further lost to breast feeding [13]. Total serum calcium levels usually decrease in accordance with pregnancy and thereafter return to baseline levels after a few months of confinement [10]. In this study, although we expected that serum calcium transitioned from mother to fetus, serum calcium did not decrease and hypocalcemia was not detected in mother. Thus, it was considered that the serum calcium level in the fetus might not have increased. (a)

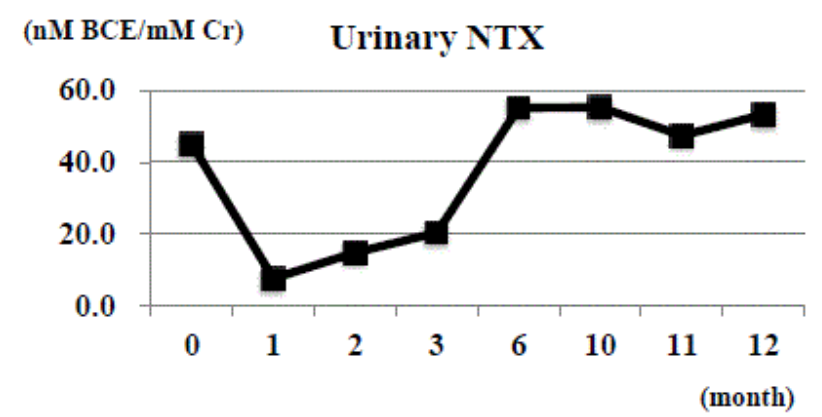

(c)

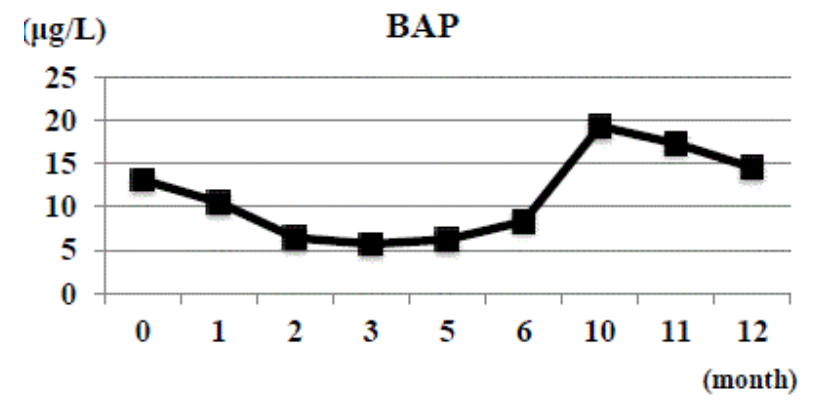

(b)

$(\mathrm{mU} / \mathrm{dL}) \quad$ TRACP-5b

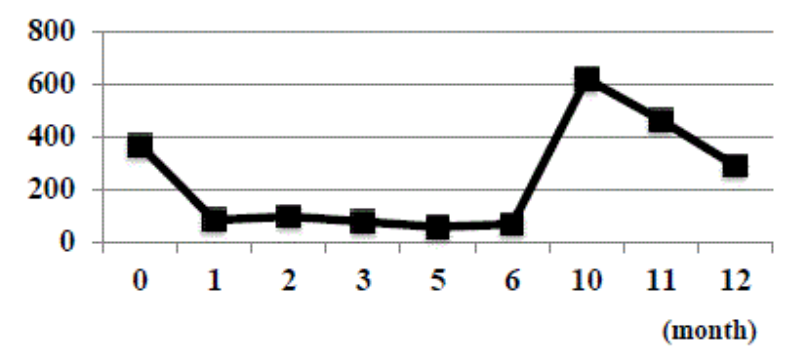

(d)

$(\mu \mathrm{g} / \mathrm{L}) \quad$ P1NP

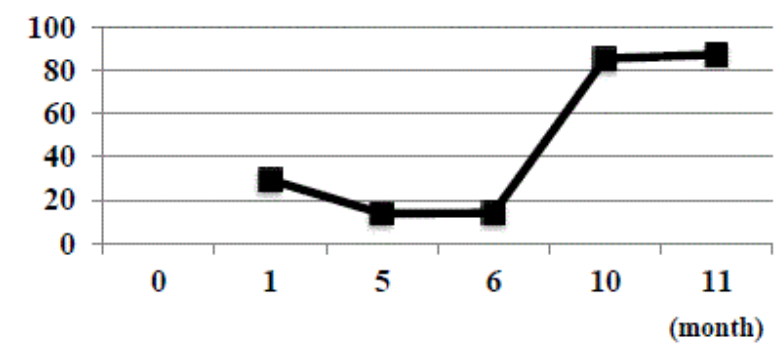

Figure 2: Changes in the values of bone turnover markers during 1 year after denosumab treatment. Urinary NTx (a) and serum TRACP-5b (b), BAP (c), and PINP (d) were measured.

Bone formation markers are transiently decreased in early pregnancy but become highly elevated in the perinatal period, while bone resorption markers tend to peak at gestation week 36 and remain high, even at the 30th week postpartum [13]. This study showed that TRACP-5b values were repressed until the second trimester and then followed expected changes [14], which suggested that denosumab exerted an effect of longer than 6 months in this patient. In support of this, whereas urinary NTx generally increases gradually during pregnancy, it remained low at half a year after denosumab administration in this study. Moreover, levels of the bone formation markers BAP and PINP were low in the first and second trimesters and high during late gestation.

$1,25(\mathrm{OH})_{2} \mathrm{D}_{3}$ increases in accordance with pregnancy to twice normal levels at late gestation and returns to baseline values within several days after delivery since prolactin, human placental lactogen, and estrogen enhance the activity of $1 \alpha-\mathrm{OH}$ ase in the kidneys [15]. The placenta might also influence $1,25(\mathrm{OH})_{2} \mathrm{D}_{3}$ increases during pregnancy to thereby enhance bone absorption from the second trimester to late gestation [16]. Here, $1,25(\mathrm{OH})_{2} \mathrm{D}_{3}$ values tended to follow a normal pattern, probably due to female hormones and the placenta, whereas $25(\mathrm{OH}) \mathrm{D}$ showed a mild increase at 8 months of pregnancy. Serum PTH does not normally change significantly during gestation [17], but decreased in early pregnancy and increased thereafter in the present case, which was potentially regulated by serum calcium levels. Serum pentosidine was decreased at 5 months, increased at months 8-11, and decreased at 12 months, and therefore may be elevated before and after delivery.

Regarding bone mass changes, Sowers et al. reported no significant differences between pre-pregnancy and post-delivery in 32 femurs in a group of 535 American women [12]. We were not able to examine the patient's bone mass immediately post-partum. Hayslip et al. described that after delivery, bone mass in lumbar vertebrae and the femur was 
Citation: Isobe F, Nakamura Y, Kamimura M, Uchiyama S, Kato H (2016) Effects of Denosumab Treatment during Early Pregnancy - A Case Report. J Nutr Disorders Ther 6: 189. doi:10.4172/2161-0509.1000189

Page 4 of 5

significantly decreased in subjects who had actively continued breast feeding, which was not seen in women who stopped breast feeding at an earlier time $[16,18]$. Yasumizu et al. found similar results for LBMD in a less than 1 month of breast-feeding group as compared with a more than 6 months breast-feeding group [19]. In this study, bone mass was increased at 1 year after denosumab treatment, suggesting that it might have risen during pregnancy due to the drug's effects.

Denosumab was given to the patient within a week of pregnancy. As the drug is believed to pass through the placenta, it may have reached the fetus to some extent to influence skeletal development. However, the endochondral ossification process starts around viviparous 8 weeks [20], while the half-life of denosumab is less than a month, suggesting that the administered denosumab would have almost completely disappeared in the mother and fetus by that time. Moreover, denosumab affects osteoclasts mostly and hence may not have altered other fetus growth. (a)

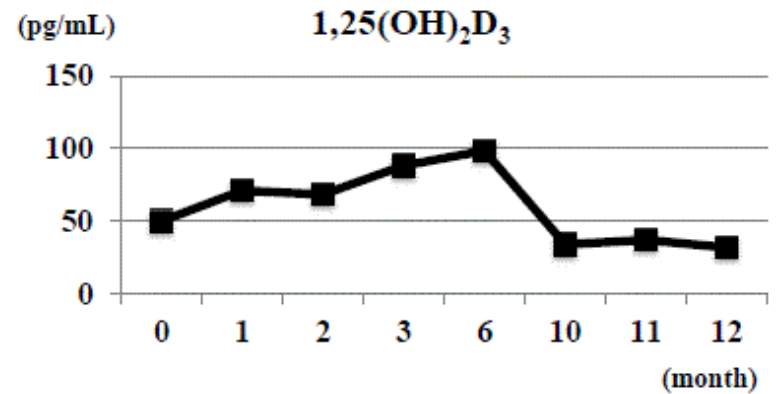

(c)

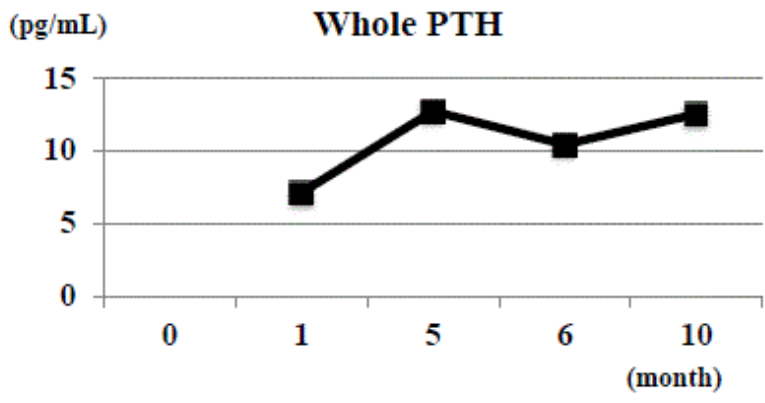

(b)

$(\mathrm{ng} / \mathrm{mL}) \quad 25(\mathrm{OH}) \mathrm{D}$

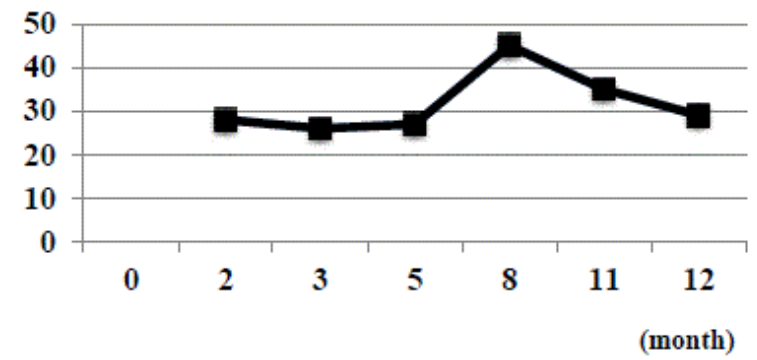

(d)

$(\mu \mathrm{g} / \mathrm{mL}) \quad$ Serum pentosidine

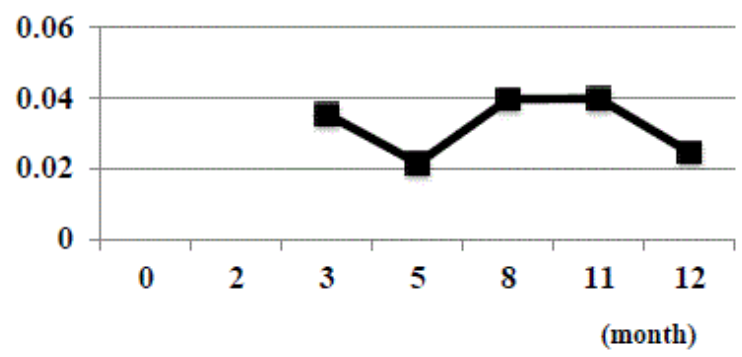

Figure 3: Changes in the values of serum $1,25(\mathrm{OH})_{2} \mathrm{D}_{3}(\mathrm{a}), 25(\mathrm{OH}) \mathrm{D}(\mathrm{b})$, Whole PTH $(\mathrm{c})$, and pentosidine (d) during 1 year after denosumab treatment.

BPs are deposited in bones over time and thereby affect bone metabolism during pregnancy. On the other hand, denosumab is an antibody, almost all of which is eliminated from the body within several months of administration. Thus, when anti-resorptive drugs for osteoporosis are required, denosumab may be safer than BPs since they exerts fewer effects on bone metabolism in pregnant women. Our results also suggest that when denosumab is administered to premenopausal women, a pregnancy test should be carried out more than once at a different time point.

In conclusion, our findings indicated that BMD and bone turnover marker values were improved at 6 months after an initial denosumab injection in a pregnant woman with osteoporosis. Whereas bone metabolism markers usually increase during late pregnancy [21], $1,25(\mathrm{OH})_{2} \mathrm{D}_{3}$ and $\mathrm{PTH}$ values were increased after only a few months in our patient, with no apparent effect on the infant.

\section{References}

1. Tanaka S, Nakamura K, Takahasi N, Suda T (2005) Role of RANKL in physiological and pathological bone resorption and therapeutics targeting the RANKL-RANK signaling system. Immunol Rev 208: 30-49.

2. Rinotas V, Niti A, Dacquin R, Bonnet N, Stolina M, et al. (2014) Novel genetic models of osteoporosis by overexpression of human RANKL in transgenic mice. J Bone Miner Res 29: 1158-1169.

3. Boyce RW, Varela A, Chouinard L, Bussiere JL, Chellman GJ, et al. (2014) Infant cynomolgus monkeys exposed to denosumab in utero exhibit an osteoclast-poor osteopetrotic-like skeletal phenotype at birth and in the early postnatal period. Bone 64: 314-325.

4. Kong YY, Yoshida H, Sarosi I, Tan HL, Timms E, et al. (1999) OPGL is a key regulator of osteoclastogenesis, lymphocyte development and lymphnode organogenesis. Nature 397: 315-323.

5. French AE, Kaplan N, Lishner M, Koren G (2003) Taking bisphosphonates during pregnancy. Can Fam Physician 49: 1281-1282. 
Citation: Isobe F, Nakamura Y, Kamimura M, Uchiyama S, Kato H (2016) Effects of Denosumab Treatment during Early Pregnancy - A Case Report. J Nutr Disorders Ther 6: 189. doi:10.4172/2161-0509.1000189

Page 5 of 5

6. Green SB, Pappas AL (2014) Effects of maternal bisphosphonate use on fetal and neonatal outcomes. Am J Health Syst Pharm 71: 2029-2036.

7. Sanz SL, García-Pérez MÁ, Tarín JJ, Cano A (2015) Bone metabolic changes during pregnancy: a period of vulnerability to osteoporosis and fracture. Eur J Endocrinol 172: R53-65.

8. Chamarthi B, Greene MF, Dluhy RG (2011) Clinical problem-solving. A problem in gestation. 365: 843-848.

9. Kovacs CS, Kronenberg HM (1997) Maternal-fetal calcium and bone metabolism during pregnancy, puerperium, and lactation. Endocr Rev 18: 832-872.

10. Seki K, Makimura N, Mitsui C, Hirata J, Nagata I (1991) Calciumregulating hormones and osteocalcin levels during pregnancy: a longitudinal study. Am J Obstet Gynecol 164: 1248-1252.

11. Delvin EE, Arabian A, Glorieux FH, Mamer OA (1985) In vitro metabolism of 25-hydroxycholecalciferol by isolated cells from human decidua. J Clin Endocrinol Metab 60: 880-885.

12. Sowers M, Corton G, Shapiro B, Jannausch ML, Crutchfield M, et al. (1993) Changes in bone density with lactation. JAMA 269: 3130-3135.

13. Kaji T, Yasui T, Sauo M (2007) Effect of bed rest during pregnancy on bone turnover markers in pregnant and postopartum women. Bone 40: 1088-1094.

14. Haruna M, Fukuoka H (1996) Metabolic turnover of bone during pregnancy and puerperium. Bull Phys Fitness Res Institute 91: 109-115.
15. Fukuoka H, Haruna M (2003) Calcium and bone metabolism during pregnancy and lactation. Clin Calcium 13: 1425-1431

16. Zerwekh JE, Breslau NA (1986) Human placental production of 1 alpha, 25-dihydroxyvitamin D3: biochemical characterization and production in normal subjects and patients with pseudohypoparathyroidism. J Clin Endcrinol Metab 62: 192-196.

17. Manabe M, Kagiya A, Tandoh T, Echizenya N, Sagara M, et al. (1996) Changes in bone mineral content and bone metabolism during pregnancy and puerperium. Nihon Sanka Fujinka Gakkai Zasshi 48: 399-404.

18. Hayslip CC, Klein TA, Wray HL, Duncan WE (1989) The effects of lactation on bone mineral content in healthy postpartum women. Obstet Gynecol 73: 588-592.

19. Yasumizu T, Nakamura Y, Hoshi K, Iijima S, Asaka A (1998) Bone metabolism after human parturition and the effect of lactation: longitudinal analysis of serum bone-related proteins and bone mineral content of lumbar spine. Endocr J 45: 679-686.

20. Rigoglio NN, Alcantara D, de Oliveira E Silva FM (2014) Endochondral ossification of the Humerus in Canine Fetuses. Microscopy.

21. Hellmeyer L, Ziller V, Anderer G, Ossendorf A, Schmidt S, et al. (2006) Biochemical markers of bone turnover during pregnancy : a longitudinal study. Exp Clin Endocrinol Diabetes 114: 506-510. 